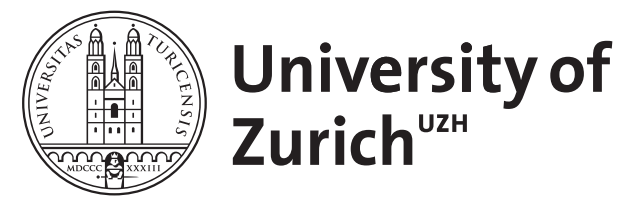

\title{
Immunotherapy for glioblastoma: quo vadis?
}

Weller, Michael ; Le Rhun, Emilie

\begin{abstract}
Glioblastoma remains essentially incurable, and new therapeutic approaches are urgently needed. Now, the findings of three serial tissue-based studies suggest that immune-checkpoint inhibition can modify the glioblastoma microenvironment. Following these encouraging observations, the results of two phase III trials of immune-checkpoint inhibition in newly diagnosed glioblastoma, with larger cohorts of patients, are eagerly anticipated.
\end{abstract}

DOI: https://doi.org/10.1038/s41571-019-0195-3

Posted at the Zurich Open Repository and Archive, University of Zurich

ZORA URL: https://doi.org/10.5167/uzh-176134

Journal Article

Accepted Version

Originally published at:

Weller, Michael; Le Rhun, Emilie (2019). Immunotherapy for glioblastoma: quo vadis? Nature Reviews. Clinical Oncology, 16(7):405-406.

DOI: https://doi.org/10.1038/s41571-019-0195-3 


\section{CNS cancer}

\section{Immunotherapy for glioblastoma: quo vadis?}

\section{Michael Weller and Emilie Le Rhun}

Refers to Zhao, J. et al. Immune and genomic correlates of response to anti-PD-1 immunotherapy in glioblastoma. Nat. Med. http://dx.doi.org/10.1038/s41591-019-0349-y (2019) | Schalper, K. A. et al. Neoadjuvant nivolumab modifies the tumor immune microenvironment in resectable glioblastoma. Nat. Med. http://dx.doi.org/10.1038/s41591-018-0339-5 (2019) | Cloughesy, T. F. et al. Neoadjuvant anti-PD-1 immunotherapy promotes a survival benefit with intratumoral and systemic immune responses in recurrent glioblastoma. Nat. Med. http://dx.doi.org/10.1038/s41591-018-0337-7 (2019).

\section{Standfirst | [Au: Please provide a brief (50-word) mini-abstract summarizing the main messages of this article]}

\section{[Au: As an alternative, perhaps you could adapt the following suggested standfirst?]}

Glioblastoma remains essentially incurable, and new therapeutic approaches are urgently needed. Now, the findings of three serial tissue-based studies suggest that immunecheckpoint inhibition can modify the glioblastoma microenvironment. Following these encouraging observations, the results of two phase III trials of immune-checkpoint 
inhibition in newly diagnosed glioblastoma with larger cohorts of patients are eagerly anticipated.

Gliomas are intrinsic brain tumours thought to arise from neuroglial progenitor cells that are highly infiltrative, often angiogenic and essentially incurable. Glioblastoma, the most common variant, is defined as a glial tumour with certain histological characteristics including necrosis and vascular proliferation. Moreover, there are typical molecular features found in subsets of glioblastomas, such as gain of chromosome 7 and loss of chromosome 10, EGFR amplifications, and telomerase reverse transcriptase promoter mutations. The vast majority of glioblastomas harbour genetic alterations in the PI3K, retinoblastoma and/or p53 signalling pathways. $^{1}$

The standard of care for patients with newly diagnosed glioblastoma consists of neurosurgical resection, as safely feasible, followed by involved field radiotherapy with concomitant and maintenance temozolomide chemotherapy. At recurrence, second surgery and re-irradiation are both options for some patients, but most patients will receive second-line systemic chemotherapy with either alkylating agents, such as lomustine, or bevacizumab. ${ }^{2}$ Median survival durations of patients receiving these treatments remain in the range of 1 year according to registry-based epidemiological studies. Numerous attempts to improve these outcomes by adding novel systemic agents to the standard of care in the first-line setting, including a phase III trial exploring the efficacy of a vaccine targeting EGFR variant III, exhibiting loss of exons 2-7, have failed to improve overall survival. ${ }^{3}$ Furthermore, the first phase III trial involving immune- 
checkpoint inhibitors in neuro-oncology, CheckMate 143 (NCT02017717), in which the efficacy of nivolumab was compared with that of bevacizumab in patients with glioblastoma at first relapse, did not demonstrate superiority of nivolumab in the overall patient population. ${ }^{4}$ The results of this trial are of continued interest because interpretation of the outcomes is dependent on whether bevacizumab alone is considered an active agent in this setting - this has never been formally tested. Furthermore, ongoing subgroup analyses of data from this trial might aid in identifying patients who are more likely, or less likely, to derive benefit from either nivolumab or bevacizumab. However, the recognition of glioblastoma as the paradigmatic lymphocyte-depleted cancer ${ }^{5}$ has further dampened the great expectations that immunotherapy might provide a therapeutic breakthrough in this disease. To date, demonstrations of clinical benefit from immunecheckpoint inhibition in patients with glioblastoma have been limited to individual patients whose tumours have excessively high mutational burdens owing to mismatch repair deficiencies.

This scenario provided the framework for three recent studies published in Nature Medicine, in which investigators attempted to identify predictors of benefit from immune-checkpoint inhibition and whether these agents have any measurable level of biological activity in patients with glioblastomas. ${ }^{6-8}$

Zhao and colleagues ${ }^{6}$ attempted to distinguish responders to immune-checkpoint inhibition from nonresponders. Following genomic and transcriptomic analyses, these investigators proposed that phosphatase and tensin homolog (PTEN) mutations are linked 
with immunosuppressive gene-expression signatures and resistance to immune-

checkpoint inhibition, whereas tumours from responders were found to harbour mitogenactivated protein kinase (MAPK) pathway alterations.

Schalper and colleagues ${ }^{7}$ adopted a pharmacodynamic approach, often referred to as a phase 0 study, in which they administered a single presurgical (at 2 weeks \pm 3 days prior to surgery) dose of nivolumab to 30 patients, the majority of whom $(n=27)$ had recurrent disease. The evolution of the tumour microenvironment of these patients was then compared to that of a reference cohort that was not exposed to immune-checkpoint inhibitors. The authors reported that nivolumab induced increased expression of several chemokine transcripts, promoted immune-cell infiltration and augmented $\mathrm{T}$ cell receptor clonal diversity in the tumour-infiltrating T cell compartment.

Data from these two studies ${ }^{6,7}$ seem to demonstrate that the glioblastoma TME is indeed not completely refractory to the immunomodulatory effects of immune-checkpoint inhibition. However, one might still argue that clinical benefit from immune-checkpoint inhibition, as defined by Zhao et al. ${ }^{6}$, remains controversial and that the historical control group presented by Schalper et $a l .{ }^{7}$ leaves doubts as to whether or not the effects on the microenvironment are specifically mediated by nivolumab; moreover, these studies, by design, do not provide evidence of clinical benefit. These various shortcomings are largely overcome by the data reported by Cloughesy et al. ${ }^{8}$ who conducted a small, randomized phase $0 / 2$ study in which patients with recurrent glioblastoma who were considered candidates for second surgery received pembrolizumab. Patients were 
randomly assigned to receive either adjuvant pembrolizumab alone or a single presurgical dose of pembrolizumab (14 \pm 5 days prior to surgery) followed by adjuvant pembrolizumab. When compared with the adjuvant only group, presurgical pembrolizumab resulted in the induction of a T cell-related and IFN $\gamma$-related gene expression signature with clonal $\mathrm{T}$ cell expansion, demonstrating that the glioblastoma TME can potentially be modified even by a single dose of pembrolizumab. Unexpectedly, patients who had been randomly assigned to receive a single presurgical dose of pembrolizumab also had prolonged overall survival durations (13.7 months versus 7.5 months, HR $0.43,95 \%$ CI $0.20-0.90 ; P=0.03)$. Imbalances in patient characteristics, if they had any effect, would have favoured the adjuvant only group because $69 \%$ of patients in this group had $\mathrm{O}^{6}$-methylguanine DNA methyltransferase (MGMT) promoter methylation as opposed to $38 \%$ in the presurgical group. Admittedly, this was a small study $(n=32)$ in which overall survival was a secondary or exploratory end point. Yet, this observation of significantly longer overall survival after a single presurgical dose of pembrolizumab encourages speculation regarding whether the iatrogenic trauma of surgery facilitates a durable immune response once the immunosuppressive properties of glioblastoma are counterbalanced by immunecheckpoint inhibition. Future studies with similar designs, potentially even involving combinations of immune-checkpoint inhibitors, are likely to follow.

Meanwhile, two phase III randomized prospective trials exploring the activity, safety and tolerability of nivolumab in patients with newly diagnosed glioblastoma have completed patient enrollment, although data are yet to be analysed. CheckMate 498 (NCT02617589) 
took advantage of the emerging concept that temozolomide can be omitted from the firstline treatment of patients with MGMT promoter-unmethylated glioblastoma owing to only marginal benefits of this approach in this patient population. ${ }^{9}$ Accordingly,

CheckMate 498 is designed to compare the efficacy of radiotherapy plus nivolumab with that of radiotherapy plus temozolomide as the current standard of care in these patients.

The companion trial, CheckMate 548 (NCT02667587), is a placebo-controlled trial designed to explore the efficacy of the addition of nivolumab to standard-of-care chemoradiotherapy in patients with MGMT-promoter-methylated glioblastoma. A concern remains that the combination of a cytotoxic agent with an immune-checkpoint inhibitor might abrogate any activation of the $\mathrm{T}$ cell compartment and could thus inhibit any relevant anti-tumour activity. However, data from animal models indicate that part of the antitumour activity of temozolomide might indeed be immune-mediated ${ }^{10}$ and chronic exposure to temozolomide might also deplete the regulatory $\mathrm{T}$ cell pool. These considerations, plus the assumption, as yet unproven, that prolonged treatment with temozolomide steadily increases the tumour mutational burden of patients with newly diagnosed MGMT-methylated glioblastoma, supports the notion that CheckMate 548 may provide the first positive phase III data from a trial involving immunotherapy in patients with brain tumours. The community will know soon.

Michael Weller is at the Department of Neurology, University Hospital and University of Zurich, Frauenklinikstrasse 26, 8091 Zurich, Switzerland. 
Emilie Le Rhun is at CHU Lille, Neuro-oncology, General and Stereotaxic Neurosurgery Service, F-59000 Lille, France.

Correspondence to M.W.

michael.weller@usz.ch

\section{References}

1 Weller, M. et al. Glioma. Nat. Rev. Dis. Prim. 1, 15017 (2015).

2 Weller, M. et al. EANO guideline on the diagnosis and treatment of adult astrocytic and

Formatiert: Englisch (Vereinigte Staaten) oligodendroglial gliomas. Lancet Oncol. 18, e315-e329 (2017).

3 Weller, M. et al. Rindopepimut with temozolomide for patients with newly diagnosed, EGFRvIII-expressing glioblastoma (ACT IV): results of a randomized, double-blind, international phase 3 trial. Lancet Oncol. 18, 1373-1385 (2017).

4 Reardon, D.A. et al. Randomized phase 3 Study evaluating the efficacy and safety of nivolumab vs bevacizumab in patients with recurrent glioblastoma: CheckMate 143.

Neuro-Oncol. 19, suppl_3, iii21 (2017).

5 Thorsson, V. et al. The immune landscape of cancer. Immunity 48, 812-830 (2018).

6 Zhao, J. et al. Immune and genomic correlates of response to anti-PD-1 immunotherapy in glioblastoma. Nat. Med. In press (2019).

7 Schalper, K.A. et al. Neoadjuvant nivolumab modifies the tumor immune microenvironment in resectable glioblastoma. Nat. Med. In press (2019).

8 Cloughesy, T.F. et al. Neoadjuvant anti-PD-1 immunotherapy promotes a survival benefit with intratumoral and systemic immune responses in recurrent glioblastoma. Nat. Med. In press (2019). 
9 Weller, M. Where does MGMT promoter methylation assessment place temozolomide in the future standards of care for glioblastoma? Cancer 124, 1316-1318 (2018).

10 Weiss, T. et al. NKG2D-dependent anti-tumor effects of chemotherapy and radiotherapy against glioblastoma. Clin. Cancer Res. 24, 882-895 (2018).

\section{Competing interests}

M.W. has received research grants from Abbvie, Adastra, Dracen, Merck, Sharp \& Dohme (MSD), Merck (EMD), Novocure, OGD2, Piqur and Roche, honoraria for lectures, participation in advisory boards and/or consultancy from Abbvie, Basilea, Bristol Meyers Squibb, Celgene, Merck, Sharp \& Dohme (MSD), Merck (EMD), Novocure, Orbus, Roche and Tocagen. E.L.R. has received research grants from Amgen and Mundipharma and honoraria for lectures or participation in advisory boards from Abbvie, Daiichi, Mundipharma, Novartis and Sankyo. 